\title{
Three Problematic Issues from a Geriatric Point of View: Cancer, Radiotherapy, and Malnutrition
}

\author{
(1) Zümrüt Bahat ${ }^{1}$, (1) Nezahat Müge Çatıkkaş² \\ ${ }^{1}$ Karadeniz Technical University Faculty of Medicine, Department of Radiation Oncology, Trabzon, Turkey \\ 2Istanbul University, Istanbul Faculty of Medicine, Department of Internal Medicine, Division of Geriatrics, Istanbul, Turkey
}

\begin{abstract}
Cancer management in older patients has become an important public health concern due to the aging population and increased incidence of cancer with advanced age. The treatment decision for older individuals should not be constrained due to their advanced chronological age. Integration of an oncogeriatric approach is a major key point to improve treatment outcomes in older patients with cancer. Thus, comprehensive geriatric assessment should be used to evaluate older patients with cancer. Malnutrition (MN) is common in patients with cancer and has a profound effect on treatment outcomes. Therefore, assessing patients' nutritional status and providing individualized nutritional intervention are essential. Radiotherapy (RT) is one of the most frequently used and effective treatment modalities against cancer in older adults and is a well-defined cause of MN. We herein highlight geriatric assessment requirements, including essential nutritional assessment in older patients with cancer. In addition to the general view, we focused on RT-related MN and its consequences. RT is better tolerated than surgery in older adults, but it may cause dehydration and MN due to RT-related diarrhea and mucositis. The clinicians should consider that in addition to its effect on the general clinical status, functionality, and surgical outcomes, the prognosis of RT is the worst in older individuals with MN.
\end{abstract}

Keywords: Assessment, cancer, geriatrics, malnutrition, radiotherapy

\section{Introduction}

Malignant neoplastic diseases are the most common cause of death after cardiovascular diseases. The incidence of cancers has been increasing globally $(1,2)$. In 2020, there were approximately 19.3 million new cancer cases, including non-melanoma skin cancer worldwide. Nearly 10 million of these cases resulted in cancer-related death (3).

In Western countries, older people constitute one-fourth of the population. Older people in the community will increase in the coming years. It is predicted that in the next fifty years, the number of people aged 65 and over will be more than double, and over the age of 85 will be almost quadruple. Cancer, an indicator of cellular senescence, is more common with aging. About $60 \%$ of new cancer cases and $70 \%$ of cancer-related deaths are seen in those aged 65 years and over. Therefore, in the future, older cancer patients will represent a global health problem resulting in increased cancer incidence and mortality $(1,4-6)$. New treatment modalities are being developed to improve the cancer-related prognosis.

Radiotherapy (RT) is an important therapeutic option in the treatment of cancers. It is applied for curative, palliative, or supportive intend in about $50-70 \%$ of cancer patients. It is an integral component of cancer treatment, and it is sometimes considered the only treatment option in cases where surgery and/or chemotherapy are risky/contraindicated in older patients. RT is a therapeutic modality applied in older adults considering their evaluation with a geriatric assessment (1). Older patients, as well as younger patients, may benefit from RT because provided that acute and late tolerance connected to RT is acceptable. Ideally, older patients should be evaluated with a comprehensive geriatric assessment (CGA) before initiation of therapy and then regularly.

Address for Correspondence: Nezahat Müge Çatıkkaş, Istanbul University, Istanbul Faculty of Medicine, Department of Internal Medicine, Division of Geriatrics, Istanbul, Turkey

Phone: +90 2124142000 E-mail: nz444mg@hotmail.com ORCID: orcid.org/0000-0003-2494-1625

Received: 13.11.2021 Accepted: 02.12.2021

Cite this article as: Bahat Z, Çatıkkaş NM. Three Problematic Issues from a Geriatric Point of View: Cancer, Radiotherapy, and Malnutrition.

Eur J Geriatr Gerontol 2022;4(1):1-4

๑Copyright 2022 by the Academic Geriatrics Society / European Journal of Geriatrics and Gerontology published by Galenos Publishing House. 
Considering the aging population and the increased incidence of cancers, the integration of the oncogeriatric approach into multidisciplinary decision processes is necessary (7). In older patients, treatment decisions should be made based on the performance score (PS), functional capacity, comorbid diseases, accompanying geriatric syndromes, frailty, medications, nutritional status, and the existing social support level. Older individuals can be managed with different treatment options, i.e., only RT, chemotherapy, surgery, their variable combinations, or palliative approach instead of a curative treatment (8). Kurtman et al. (9) drew attention to the characteristics of the tumor, accompanying risks, functional capacity, palliative need, and benefit/harm in RT applications in older patients. They underlined that more palliative approaches could be considered in older patients with low PS and functional capacity, high comorbidity score, poor nutritional status, and other poor prognostic factors (10).

In clinical practice, older patients are being frequently undertreated even when they do not have any other disease or functional problem just due to the consideration of their chronological age. For example, treatment choice has been stratified by only age in some cases; i.e., $\geq 80$ years were directed to less intensive treatment $(8,9)$. Obviously, the treatment decision of an older individual should not be constrained due to an advanced chronological age $(8,9)$. Some studies reported that mortality and morbidity for different tumor types are similar for older and younger patients (10). Some comorbidities that may already be present in older adults may worsen treatment outcomes; nevertheless, the treatment plan should not be made just due to the presence of comorbidities. In this context, there is not any data that can justify and validate age discrimination that is being made during the treatment of older cancer patients, which can excuse the administration of less effective treatments to older patients (10).

Comprehensive geriatric assessment (CGA) accepted as the standard for estimating life expectancy, morbidity, and mortality in cancer patients aged 70 years and over $(1,2)$. Carnofsky performance status and Eastern Cooperative Oncology Group performance measures are associated with the treatment toxicity; nevertheless, these scales alone cannot predict outcomes as successful as CGA in the older population $(1,2)$. There are very few studies in which the geriatrician and oncologist have worked together to manage the treatment plan. In the ELCAPA study, geriatricians made recommendations that may modify the treatment plan in older cancer patients through CGA. Subsequently, 20.8\% of the patients required a reduction in the treatment intensity (11). In a study by Mohile et al. (6), 161 patients (57 men, 104 women; median age 82.4 years), 50\% of whom had advanced cancer, were involved and referred for CGA at a university hospital. After the discussion at the oncogeriatric multidisciplinary consultation, cancer treatment was changed in 79 (49\%) patients, including delayed treatment in 5 patients, less intensive treatment in 29 patients, and more intensive treatment in 45 patients. Horgan et al. (12) reported that eligible older people are not referred to geriatric evaluation, while a geriatric assessment might have changed the initial treatment decision. On the other hand, clinicians need controlled studies to determine whether the treatment modifications based on CGA can improve treatment outcomes. Implementation of multidisciplinary expertise, including social workers, physical therapists, occupational therapists, and nutritionists, can help develop CGA-guided interventions in older individuals with cancer. Accordingly, the risks can be identified, and a CGA-guided treatment plan can be determined. As a simple example, a geriatrician or a pharmacologist can assess the currently used medications, correct the inappropriate medication use, and thereby reduce the potential side effects due to medications.

Nutrition is one of the major issues to be evaluated during the oncologic assessment (13). Malnutrition (MN) results from a deficiency in energy, protein, and micronutrient intake, causes changes in body composition and adversely affects the patient's bodily functions and clinical status $(14,15)$. It has been reported that, in many cancers, $15-40 \%$ of the patients had already lost weight and become malnourished before the initiation of the treatment (13). Moreover, the MN incidence may increase further during the treatment of cancer. Specifically, considering $\mathrm{RT}$, the prevalence of MN is estimated between 15-80\% during RT application $(13,15)$. This rate is higher in some types of cancers treated with RT, such as head and neck, esophagus and other gastrointestinal system, pancreaticobiliary, and lung cancers (13-15). While RT is tolerated better than surgery in older population, the risk of dehydration due to RT-related diarrhea and mucositis and the resulting MN status should be carefully considered $(7,16)$. Consequent cachexia developing in cancer settings directly brings about $20 \%$ of all cancer-related deaths $(2,6)$.

\section{Diagnosis of Malnutrition in Older Cancer Patients, Including Those Treated with Radiation Therapy}

Considering the risk of $\mathrm{MN}$ in cancer patients, it is essential for oncologists to assess the patients' nutritional status and provide individualized nutritional intervention promptly to optimize clinical outcomes. In this regard, oncologists should evaluate the patients' nutritional status before treatment, determine the potential risk factors that may lead to weight loss, and detect the risk of MN immediately. Thus, MN should be screened and detected in the early reversible phase before refractory cachexia occurs $(14,15)$. Previous studies noted that the weight loss was lesser when a nutritional evaluation and management was performed before, during, and after cancer treatment $(2,14)$. Consequently, the clinicians can aid in the maintenance and/ 
or improvement of mental and physical functions, reduction of treatment and disease-related complications, decreasing disease severity, lower use of resources, and shortened hospitalization duration (14).

If an older patient has weight loss ( $>5 \%$ in six months or $>10 \%$ over six months) or has a markedly reduced body mass index (BMI) (i.e., BMl $<20 \mathrm{~kg} / \mathrm{m}^{2}$ ), or involuntary loss of muscle mass, she/he should be considered having $\mathrm{MN}$ symptoms and signs. $\mathrm{MN}$ can be defined and recognized by variable definitions. The most recent definition is the global consensus approach [global leadership initiative on malnutrition (GLIM)] for the diagnosis of MN. Global leadership initiative on malnutrition (GLIM) suggests that the diagnosis of $\mathrm{MN}$ is made when there is at least one phenotypic criterion (i.e., involuntary weight loss, low $\mathrm{BMI}$, or decreased muscle mass) and one etiological criterion (i.e., reduced food intake/malabsorption or acute/chronic disease-related inflammation) (2,8). 2021 updated European Society of Parenteral and Enteral Nutrition Guidelines for clinical nutrition in cancer patients recommended the use of the following scales for the assessment of nutrition in cancer patients: Nutritional risk screening-2002, malnutrition universal screening tool (MUST), mini nutritional assessment (MNA), and malnutrition screening tool (MST). The Academy of Nutrition and Dietetics recommends using of MST and MUST (17). Among these evaluation methods, MUST (in the outpatient setting) and MNA (in both outpatient and inpatient settings) are preferred in older cancer patients $(2,14,15)$. Global leadership initiative on malnutrition (GLIM) definition and evaluation can be preferred in older adults as well. Obviously, the diagnostic approach to detect $\mathrm{MN}$ does not differ according to the chosen treatment modality, including RT.

\section{Causes of Malnutrition in Older Cancer Patients, Including Those Treated with Radiation Therapy}

Patient-specific, disease-specific, and treatment-specific factors may lead to MN. Radiotherapy (RT) generally has side effects specific to the region where it is applied, and adverse nutritional effects appear due to these side effects.

The patient and disease-specific factors include poor pretreatment nutritional status, current micronutrient, antioxidant, and mineral deficiencies, low BMI, poor PS, advanced age, caucasian race, female gender, genetic polymorphisms, comorbidities, smoking, fatigue, weight loss, loss of appetite, anorexia, anxiety, depression, dyspnea, pain, advanced cancer, type of cancer, localization of cancer, gastrointestinal system obstruction, impaired absorption of nutrients, metabolic changes due to hormones secreted in cancers, immune-activation via the released cytokines, insulin resistance that develops as a result of the inflammatory process, increased catabolism associated with cancers, and low prealbumin and albumin levels $(2,13-15,18)$.
Treatment-specific factors that may cause $\mathrm{MN}$ are anorexia, fatigue, surgery, chemotherapy, and RT-related systemic and local toxicities, mucositis, xerostomia, dysphagia, esophagitis, diarrhea, enteritis, nausea/vomiting, hematological side effects, anxiety, depression, micronutrient, antioxidant, and mineral deficiencies, taste changes and the intend and extend of the treatment (curative, palliative, external, brachytherapy, stereotactic body radiation therapy, etc.). Radiotherapy-related treatment toxicity augments, especially when it is applied with concurrent chemotherapy. These may lead to weight loss and MN by reducing nutrient intake $(13,15,18$ 20). Identifying and treating the side effects that may affect the nutritional status of older patients receiving treatments, including $\mathrm{RT}$, will reduce the possibility of interrupting the therapy and have the capacity to improve tumor control.

\section{Consequences of Malnutrition in Older Cancer Patients with a Specific Emphasis on Those Treated with Radiation Therapy}

Malnutrition (MN) has been shown to be associated with a decreased functional capacity due to muscle loss, augmentation of symptoms, decreased quality of life and treatment tolerance, worse prognosis, and reduced survival $(13,15)$. On the other hand, $\mathrm{MN}$ causes the progression of many diseases that may be simultaneously present in the patient other than cancer, and it is a unique factor in the development of sarcopenia, cachexia, and frailty, which are themselves associated with increased morbidity and mortality (8). Malnutrition (MN) may cause adverse alterations in absorption, protein binding, hepatic metabolism, and renal excretion of drugs and their metabolites during systemic therapies. In malnourished patients, decreased plasma protein concentration may significantly increase the likelihood of toxicity of agents with high protein binding, such as prednisolone, etoposide, cisplatin, paclitaxel, and irinotecan metabolites. In addition, MN may disrupt some metabolic pathways in the liver, reducing the clearance of drugs and prolonging their half-life. Thus, it has been claimed that since patients will be exposed to more drugs, toxicity may increase, and the necessity to interrupt the treatment will occur more frequently in patients suffering concomitantly from MN (15).

Malnutrition (MN) influences the patient's tolerance to the treatment. Weight loss indicates that the patient may not tolerate a curative treatment, complete the treatment, thereby can not receive adequate treatment, and, hence, weight loss increases mortality risk $(21,22)$. Specifically, the prognosis of RT is the worst in older individuals with MN. Many studies have been published reporting that $\mathrm{MN}$ had an adverse effect on treatment and decreased favorable response to the introduced treatment. Increased RT toxicity, changing, stopping, or interrupting the RT plan may be negative factors that change the use of a curative or palliative procedure and decrease the response to RT, especially when combined with chemotherapy $(13,15)$. 
Another point is that MN escalates surgical morbidity and post-surgical complications. It can delay wound healing, worsen muscle functions, and increase the risk of postoperative complications, thus prolonging hospital stay and increasing the financial burden (15).

\section{Conclusion}

The integration of the oncogeriatric approach is a major key point to improve outcomes in older cancer patients. Malnutrition (MN) is common in the cancer setting and has a profound effect on treatment outcomes. It is essential to assess the patients' nutritional status generally via MUST or MNA and provide individualized nutritional intervention. Radiotherapy (RT) is one of the most frequently used and effective treatment modalities in older adults. While RT is tolerated better than surgery in older adults, it is a well-defined cause of MN. The clinicians should consider that, in addition to its effect on general clinical status, functionality, and surgical outcomes, the prognosis of RT is the worst in older individuals with MN as well.

\section{Acknowledgments}

The authors certify that they comply with ethical guidelines for authorship and publishing of the European Journal of Geriatrics and Gerontology.

\section{Ethics}

Peer-review: Externally peer-reviewed.

\section{Authorship Contributions}

Surgical and Medical Practices: Z.B., N.M.Ç., Concept: Z.B., Design: Z.B., Data Collection or Processing: Z.B., N.M.Ç., Analysis or Interpretation: Z.B., N.M.Ç., Literature Search: Z.B., N.M.Ç., Writing: Z.B., N.M.Ç.

Conflict of Interest: No conflict of interest was declared by the authors.

Financial Disclosure: The authors declared that this study received no financial support.

\section{References}

1. Muelas Soria R. [Nutritional impact of radiotherapy in oncogeriatric patients. Can radiation oncologists minimize the radiotherapy impact on the nutritional status of the elderly patient?]. Nutr Hosp 2020;37:1.

2. Muscaritoli $M$, Arends J, Bachmann $P$, Baracos V, Barthelemy N, Bertz $H$,

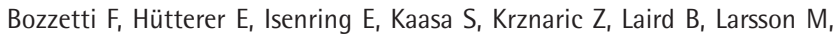
Laviano A, Mühlebach S, Oldervoll L, Ravasco P, Solheim TS, Strasser F, de van der Schueren M, Preiser JC, Bischoff SC. ESPEN practical guideline: Clinical Nutrition in cancer. Clin Nutr 2021;40:2898-2913.

3. Sung $H$, Ferlay J, Siegel RL, Laversanne $M$, Soerjomataram I, Jemal A, Bray F. Global Cancer Statistics 2020: GLOBOCAN Estimates of Incidence and Mortality Worldwide for 36 Cancers in 185 Countries. CA Cancer J Clin $2021 ; 71: 209-249$
4. American Cancer Society. Cancer Treatment \& Survivorship Facts \& Figures 2019-2021. Atlanta: American Cancer Society; 2019.

5. Blasiak J, Chojnacki J, Pawlowska E, Szczepanska J, Chojnacki C. Nutrition in Cancer Therapy in the Elderly-An Epigenetic Connection? Nutrients 2020;12:3366.

6. Mohile SG, Klepin HD, Rao AV. Considerations and controversies in the management of older patients with advanced cancer. Am Soc Clin Oncol Educ Book 2012:321-328.

7. Durdux C, Boisserie T, Gisselbrecht M. [Radiation therapy in elderly patients] Cancer Radiother 2009;13:609-614.

8. Volkert D, Beck AM, Cederholm T, Cruz-Jentoft A, Goisser S, Hooper L, Kiesswetter E, Maggio M, Raynaud-Simon A, Sieber CC, Sobotka L, van Asselt D, Wirth R, Bischoff SC. ESPEN guideline on clinical nutrition and hydration in geriatrics. Clin Nutr 2019;38:10-47.

9. Kurtman C, Gareveran MS, Gümüştepe E. Geriatric Radiotherapy. Ankara Eğt. Arş. Hast. Derg 2016;49:122-127.

10. Monfardini S, Balducci L, Overcash J, Aapro MS. Surgical and Radiation Oncology in Elderly Patients with Cancer October 25, 2020. The ASCO post. https://ascopost.com/issues/october-25-2020/surgical-and-radiationoncology-in-elderly-patients-with-cancer/.

11. Caillet P, Canoui-Poitrine F, Vouriot J, Berle M, Reinald N, Krypciak S, Bastuji-Garin S, Culine S, Paillaud E. Comprehensive geriatric assessment in the decision-making process in elderly patients with cancer: ELCAPA study. J Clin Oncol 2011;29:3636-3342.

12. Horgan AM, Leighl NB, Coate L, Liu G, Palepu P, Knox JJ, Perera N, Emami M, Alibhai SM. Impact and feasibility of a comprehensive geriatric assessment in the oncology setting: a pilot study. Am J Clin Oncol 2012;35:322-328.

13. Ravasco P. Nutrition in Cancer Patients. J Clin Med 2019;8:1211.

14. Reber $E$, Schönenberger KA, Vasiloglou MF, Stanga Z. Nutritional Risk Screening in Cancer Patients: The First Step Toward Better Clinical Outcome. Front Nutr 2021;8:603936.

15. Santarpia L, Contaldo F, Pasanisi F. Nutritional screening and early treatment of malnutrition in cancer patients. J Cachexia Sarcopenia Muscle $2011 ; 2: 27-35$

16. Huguenin $P$, Glanzmann $C$, Lütolf UM. Acute toxicity of curative radiotherapy in elderly patients. Strahlenther Onkol 1996;172:658-663.

17. Arends J, Bachmann P, Baracos V, Barthelemy N, Bertz H, Bozzetti F, Fearon

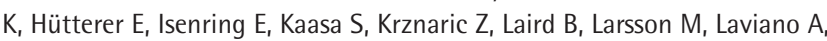
Mühlebach S, Muscaritoli M, Oldervoll L, Ravasco $P$, Solheim T, Strasser F, de van der Schueren M, Preiser JC. ESPEN guidelines on nutrition in cancer patients. Clin Nutr 2017;36:11-48.

18. Cacicedo J, Casquero F, Martinez-Indart L, del Hoyo 0 , Gomez de Iturriaga $A$, Navarro A, Bilbao P. A prospective analysis of factors that influence weight loss in patients undergoing radiotherapy. Chin J Cancerm 2014;33:204-210.

19. Donaldson SS, Lenon RA. Alterations of nutritional status: impact of chemotherapy and radiation therapy. Cancer 1979;43(5 Suppl):2036-2052.

20. Richards J, Arensberg MB, Thomas S, Kerr KW, Hegazi R, Bastasch M. Impact of Early Incorporation of Nutrition Interventions as a Component of Cancer Therapy in Adults: A Review. Nutrients 2020;12:3403.

21. Barthelemy N, Streel S, Donneau AF, Coucke P, Albert A, Guillaume M. Screening for malnutrition in lung cancer patients undergoing radiotherapy. Support Care Cancer 2014;22:1531-1536.

22. Ge T, Lin T, Yang J, Wang M. Nutritional status and related factors of patients with advanced lung cancer in northern China: a retrospective study. Cancer Manag Res 2019;11:2225-2231. 